\title{
Automated search for livestock enclosures of rectangular shape in remotely sensed imagery
}

\author{
Igor Zingman $^{a}$, Dietmar Saupe ${ }^{a}$, Karsten Lambers $^{b}$ \\ ${ }^{a}$ Department of Computer and Information Science, University of Konstanz, Germany \\ ${ }^{b}$ Institute of Archaeology, Heritage Sciences and Art History, University of Bamberg, Germany
}

\begin{abstract}
We introduce an approach for the detection of approximately rectangular structures in gray scale images. Our research is motivated by the Silvretta Historica project that aims at automated detection of remains of livestock enclosures in remotely sensed images of alpine regions. The approach allows detection of enclosures with linear sides of various sizes and proportions. It is robust to incomplete or fragmented rectangles and tolerates deviations from a perfect rectangular shape. Morphological operators are used to extract linear features. They are grouped into parameterized linear segments by means of a local Hough transform. To identify appropriate configurations of linear segments we define convexity and angle constraints. Configurations meeting these constraints are rated by a proposed rectangularity measure that discards overly fragmented configurations and configurations with more than one side completely missing. The search for appropriate configurations is efficiently performed on a graph. Its nodes represent linear segments and edges encode the above constraints. We tested our approach on a set of aerial and GeoEye-1 satellite images of $0.5 \mathrm{~m}$ resolution that contain ruined livestock enclosures of approximately rectangular shape. The approach showed encouraging results in finding configurations of linear segments originating from the objects of our interest.
\end{abstract}

Keywords: Structure detection, incomplete rectangles, graph cliques, convexity constraint, rectangularity measure.

\section{INTRODUCTION}

We present an approach for finding rectangular structures in gray scale images. The rectangular structures are of various sizes and proportions, they may be fragmented and may have one side completely missing. This work was motivated by an archaeological project that aims at automated detection of livestock enclosures occurring in Alpine regions, see Ref. 1,2. These structures are usually built of linear walls that form an approximately rectangular enclosure. Unfortunately, the structures of archaeological interest are usually ruined and appear at very low contrasts in remotely sensed images, such as the satellite or aerial images of $0.5 \mathrm{~m}$ resolution used in our project. At such a resolution, the width of the structure walls usually does not exceed two pixels. Detection of such faint structures in a complex terrain, which is characterized by various irrelevant structures and textures having much higher contrasts, is a very challenging task.

Detection of rectangular structures was previously addressed in different contexts. Here we will not give an exhaustive review of these approaches, but only mention several representative examples, some of which are related to our approach. Many methods were proposed for detection of rectangular buildings in remotely sensed images. In Ref. 3, the authors use geometric and projective constraints for generating building hypotheses from lines. The lines were extracted be means of an edge detector followed by linking and line approximation. The process of hypotheses generation involved time expensive reasoning. The hypotheses were further verified using 3D cues. Markov Random Fields (MRF) were used in Ref. 4 to delineate buildings. Spatial dependencies were specified in a probabilistic framework using the MRF model. An energy function associated with the MRF was minimized resulting in the appropriate grouping of lines. False contours and contours that were missed were not discussed, and run times were not reported in this work. In Ref. 5, a search on a directed graph was used to generate building hypotheses. Line approximations of linked edges served as nodes of a graph, while values of graph edges encoded line relations. A search for closed loops in the graph accompanied by an additional extensive set of rules was used to generate building hypotheses. A subsequent hypothesis validation was based on $3 \mathrm{D}$ cues and existence of shadows. Our approach is similar to Ref. 5 in the sense that it uses graph nodes to

Konstanzer Online-Publikations-System (KOPS)

URL: http://nbn-resolving.de/urn:nbn:de:bsz:352-249966 
represent lines and line relations are represented by graph edges. In our approach, however, we use undirected graph with non-weighted edges and the search is performed for maximal cliques. We also use a very simple reasoning based on only two rules (angle and convexity rules), which contributes to relatively small computing times of our approach. In contrast to the building detection methods, our approach does not require edge linking and line approximations with identification of the end points. This makes our approach more robust to clutter and small changes in appearance of linear features. Thus, we also avoid heuristics inherent to such stages. The building detection methods described above are hardly applicable to our task because in contrast to livestock enclosures, buildings are very distinct features. Moreover, walls of livestock enclosures are very low, may be highly fragmented, or even completely ruined. 3D cues are not available and shadows frequently do not exist or are very weak.

The Hough transform is known as a robust technique for detection of lines (or other parameterized shapes) in cluttered environments. In Ref. 6, the Hough transform was proposed for the detection of two sets of perpendicular lines in the context of rooftop polygon extraction. A general approach for detection of rectangular contours based on the Hough transform was developed in Ref. 7. In our approach, we also use the Hough transform for grouping of aligned ridge features. The authors of Ref. 7 suggest to compute a local Hough transform using a sliding window. A set of simple constraints on peaks in the Hough plane was designed to search for two orthogonal pairs of parallel lines. These constraints restrict opposite lines to be symmetric relative to the central point of a local window and to be of the similar length. In many practical applications, however, including the detection of livestock enclosures, such a basic approach with very strict rules fails to detect fragmented or incomplete structures. It may also detect line configurations that cannot form a rectangular contour. This is prevented in our approach by adding a convexity constraint. Furthermore, we do not restrict the number of sides of an enclosure to be exactly four, thus allowing incomplete rectangles and polygons with angles around either 90 or 180 degrees. Another disadvantage of the Hough based method in Ref. 7 is that the computation of the local Hough transform at each image pixel is time consuming. Though in our approach we also use local windows, we place them at carefully chosen sparse candidate points.

\section{OVERVIEW}

In our approach, we use a graph representation that encodes linear segments and their spatial relations. We suggest convexity and angle constraints applied to linear segments to identify appropriate configurations of linear segments. Such configurations, represented by graph subsets, are found by an efficient search on the graph. Nodes of the graph correspond to the linear segments and its edges encode the angle and convexity constraints described in Sec. 6. Appropriate configurations, later also termed valid configurations, are further ranked by a new rectangularity measure that emphasizes rectangular structures with at least three sides. These sides may be fragmented or incomplete. A configuration with the highest rectangularity measure is found among maximal cliques of the graph.

The block diagram of our approach is shown in Fig. 1. Blocks 4 and 5, which are central in our approach, describe novel approaches shortly summarized above. This is described in detail in Sec. 6. The flow includes the first block that generates a binary image of ridge points belonging to linear ridge features of some minimal length. The groups of aligned ridge points are further represented by parameterized linear segments in the third block (see Sec. 5). Parameters of linear segments are found in the Hough plane. The goal of the second block is to determine sparse candidate points that may be centers of configurations of linear segments possibly originating from enclosures we are looking for. In Sec. 4 we suggest a simple and fast technique that calculates the candidate points and an approximate size of a corresponding structure. The central part of our approach is represented by the forth and fifth blocks that process linear segments around each candidate point determined in the second block. As a result, each candidate point receives the rectangularity score of an optimal configuration of linear segments in the region centered at this point. Optimal configurations with the highest rectangularity measure are efficiently searched on the graph. The final map of rectangularity scores serves us as the likelihood map of existence of rectangular enclosures. 


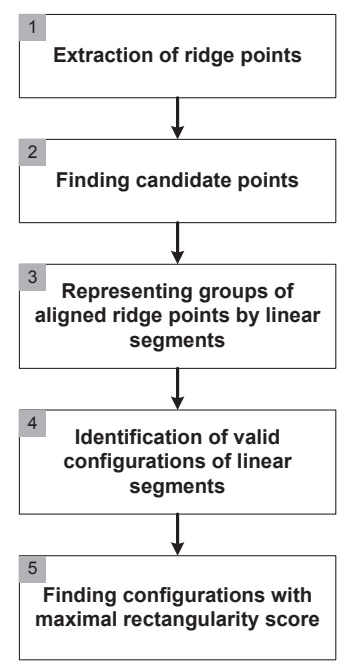

Figure 1. The principal flow used for detection of approximately rectangular enclosures. An overview is given in Sec. 2 .

\section{EXTRACTION OF RIDGE POINTS}

In this section we present our methodology for extraction of linear ridge features from an original gray tone image. This corresponds to the first block of the diagram in Fig. 1. To extract points belonging to linear ridge features, we use a technique we have recently developed in Ref. 8. It allows extraction of isolated ridge features of a predefined minimal length while suppressing background texture. This is achieved by using a Top-Hat transform followed by the Morphological Feature Contrast (MFC) operator, see Ref. 8, 9, and by a composite opening obtained by point-wise maximum of morphological openings with linear structuring elements at different orientations. Non-zero pixels of the resulting map are taken as ridge features.

Walls of livestock enclosures usually appear brighter than the background. On the other hand, shadows of walls may exist, and they usually appear at higher contrast than the walls themselves. To be sensitive to both the wall and its shadow, we apply the above technique twice for bright and dark ridge features. The latter are also called valley features. The technique in Ref. 8 also allows assignment of an orientation angle to each extracted ridge point. This angle is defined as an orientation at which an opening with a linear structuring element yields a maximum value. The second column of Fig. 4 shows examples of resulting binary maps when this technique was applied to images that contain livestock enclosures. The minimal length parameter (the length of the linear structuring element of the composite opening) was set to 15 pixels. The size of a square structuring element for the Top-Hat operator was set to 5 pixels, while the sizes of two square structuring elements for the MFC operator were set to 5 and 10 pixels.

\section{LOCALIZATION OF CANDIDATE POINTS}

In Sec. 6 we describe an approach that analyzes configurations of linear segments (see Sec. 5) within an image window centered at different points of the image. As a result, the center points will receive a value proportional to the likelihood of the existence of a rectangular enclosure in the correspondent window. There is no need to analyze image patches around each point in the image, because patches at neighboring locations contain the same linear segments. Such an analysis is also very time consuming. We have therefore developed a simple and fast procedure that identifies sparse points, around which configurations of linear segments should be further analyzed. We call these points candidate points. The size of a local analyzing window centered at candidate points, which is used in blocks 3, 4, 5 of the diagram in Fig. 1, is determined adaptively as described below.

After a binary image of ridge features is generated, we perform thinning of the image complement. This produces one pixel wide skeleton lines equally distant from the ridge features. A thinning algorithm described in Ref. 10 is used for this purpose. We sample the skeleton in order to get sparsely distributed candidate points. The sampling is performed as follows. Given a map of skeleton points, we visit them in row-wise order from left 


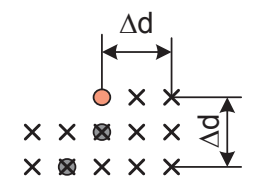

Figure 2. Sampling a skeleton. Skeleton points (marked by circles) are visited in a row-wise order from left to right and top down. Given a current skeleton point (red circle), not yet visited skeleton points (marked by gray circles) in its neighborhood (marked by crosses) are removed. The size of the neighborhood is controlled by the sampling distance $\Delta d$.

to right and top to bottom. Having visited a particular point of a skeleton we remove not yet scanned skeleton points in the neighborhood defined by the distance $\Delta d$ as illustrated in Fig. 2. We use a variable $\Delta d$ proportional to the size of analyzing window determined at each candidate point adaptively as elaborated in the following paragraph.

Along with the skeleton we compute the distance transform of the binary map of ridge features. The distance transform assigns to each pixel the distance to the nearest point in the input binary image. We use the values of the distance transform at candidate points to determine an adaptive size of an analyzing window. Particularly, we use a circular analyzing window of a radius equal $1.6 D$, where $D$ is a value of the distance transform at a corresponding candidate point*. Thus, the size of the analyzing window is proportional to the size of potential structures. To keep the number of sampled candidate points in an analyzing window of varying size approximatively constant, we sample the skeleton with variable $\Delta d$. For each point of the skeleton the sampling distance $\Delta d$ is computed as $\Delta d=2 D / n_{s p}$, where $n_{s p}$ is a constant value that determines the number of samples within an analyzing window.

Given dark and bright ridge features, we compute two images of skeleton lines and two correspondent distance transforms ${ }^{\dagger}$. To generate a single map of candidate points the union of two skeletons could be used. However, points common to both skeletons may exist with the two associated distance values from two distance transforms. This problem of the equivocal distance can be avoided using sampled skeletons. The skeleton computed from the dark ridge features is sampled first. Then, points of the second skeleton (computed from the bright ridge features) that are common to the sampled first skeleton are removed. Finally, the remaining points of the second skeleton are sampled. The resulting sampled skeletons that do not have common points are combined. This strategy allows an unequivocal assignment of the distance values from the two distance transforms to points of the combined sampled skeleton. The third column of Fig. 4 gives examples of generated candidate points (in red) computed for $n_{s p}=6$. We use this value for all the experiments shown in Sec. 7. Skeleton points with the doubled distance values smaller or greater than predefined minimal and maximal sizes of structures, respectively, are removed. In our experiments in Sec. 7, the minimal and maximal distances between parallel walls of an enclosure were set to 10 and 90 pixels, respectively.

Since the thinning and distance transform algorithms are very fast, Ref. 10,11, our approach for localization of candidates points is time efficient. Note that skeleton lines may also be computed from the distance transform by means of localization of its ridges, see for example Ref. 12,13. However, we have found that such an approach may miss some skeleton points, because these points are not always the local maxima of the distance transform and not always the local maxima in the direction of a gradient of the distance transform.

\section{LINEAR SEGMENTS}

In this section we present our methodology for parametrization of linear segments given an image of ridge points extracted in the first block of the diagram in Fig. 1. We represent a group of aligned ridge points by a triple of

\footnotetext{
* If we consider a candidate point at a rectangle center, the radius $R$ of the analyzing window should be taken equal to halve of the length of the rectangle diagonal to include all the points of the rectangle. The distance from the candidate point to the closest side of the rectangle equals $D$ known from the distance transform. Therefore, the radius $R$ of the analyzing window supports detection of rectangular structures roughly up to an aspect ratio of a rectangle equal to $\sqrt{\left(\frac{R}{D}\right)^{2}-1}$

${ }^{\dagger}$ Using a single combined map of dark and bright ridge features may result in inappropriately biased skeleton, because dark and bright features frequently belong to different structures.
} 
parameters $(\theta, r, l)$ found by means of a local Hough transform centered at candidate points. The size of local analyzing windows is adaptive and determined as described in Sec. 4. We use the Hough transform in the form introduced in Ref. 14, where a line is defined by the orientation $\theta$ of the normal and a distance $r$ from the origin

$$
r=x \cos \theta+y \sin \theta .
$$

A peak in the $(\theta, r)$ plane, also called Hough plane, corresponds to a linear segment $\mathcal{S}$, which can also be fragmented as shown in Fig. 3(a). The parameter $l$ in the triple $(\theta, r, l)$ is the number of points that belong to the linear segment and is computed as the height of the correspondent peak in the Hough plane. The peaks are found as regional maxima in the Hough plane that was discretized with $\Delta \theta=3$ degrees and $\Delta r=1$ pixel. To better relate the parameter $l$ to the length and avoid its dependence on the width of the extracted ridge features, we perform their thinning (see Ref. 10) prior to clustering in a Hough plane. The thinning reduces the width of all structures to one pixel.

Since ridge features are extracted together with their orientations, $r$ can be directly computed for each feature point $(x, y)$ using Eq. (1). Thus, each feature point votes for a single point in the $(\theta, r)$ plane instead of voting for a curve as suggested in Ref. 14. This idea, which was already used in Ref. 15 for clustering of short ridge features and in Ref. 16 for clustering of point features, considerably eases extraction of meaningful peaks in the Hough plane. This clustering technique computed in a local window can actually be considered as an extension of edge orientation histograms, which are at the core of most successful feature sets used for detection of certain object classes, Ref. 17,18. In contrast to edge orientation histograms, the $(\theta, r)$ plane based technique allows detection not only of dominant orientations of local features, but also their spatial alignment.

\section{FINDING RECTANGULAR PATTERNS}

We first describe the principles of our approach, which later in Sec. 6.1 and in Sec. 6.2 will be worked out in detail. The linear segments extracted in Sec. 5 are used as basic primitives. Our approach for detection of rectangular enclosures is based on finding valid configurations (subsets) of linear segments around a candidate point. These configurations are further scored in a way such that configurations likely to originate from a rectangular enclosure obtain a higher score. We impose two constraints on linear segments of a valid configuration:

1. Linear segments of every pair are either approximately parallel or perpendicular to each other.

2. Extension of all linear segments can form a nearly convex contour.

Given a candidate point around which we look for a valid configuration, the second constraint can be ensured by pair-wise relations between linear segments. The pair-wise relations are detailed in Sec. 6.1.

A valid configuration models not only perfect rectangles or their parts, but also convex polygons (or their parts) with angles around either 90 or 180 degrees. Livestock enclosures of our interest are frequently better modeled by such polygons than by perfect rectangles. To find valid configurations near a particular candidate point, we build a graph with nodes that correspond to linear segments. Each node is attributed by three parameters $\theta, r$, and $l$, where $\theta$ is the segment orientation, $|r|$ is the distance from the candidate point to the line on which the segment lies, and $l$ is the number of points of the segment. An edge between two nodes of a graph is set if the pair of correspondent linear segments satisfies the first and ensures the second constraint mentioned above. Valid configurations of linear segments correspond to cliques ${ }^{\ddagger}$ of this graph.

We denote linear segments by $\mathcal{S}$ and call a set of linear segments $\mathcal{S}_{i}$ with similar $r_{\mathcal{S}_{i}}$ and $\theta_{\mathcal{S}_{i}}$ a linear structure $\mathbf{L}$ with a characteristic $\theta_{L}$ and $r_{L}$, see Fig. 3(a). Due to the constraints mentioned above, a valid configuration will have at most two characteristic orientations $\theta_{L}$ that differ approximately by 90 degrees. For each $\theta_{L}$ there may be at most two characteristic $r_{L}$. Thus, at most four characteristic pairs of $\left(\theta_{L}, r_{L}\right)$ are possible that correspond to four linear structures enclosing the given candidate point. Valid configurations may correspond,

\footnotetext{
${ }^{\ddagger}$ A clique is a subgraph that is fully connected, i.e. each node of the subgraph is connected to all other nodes of this subgraph.
} 


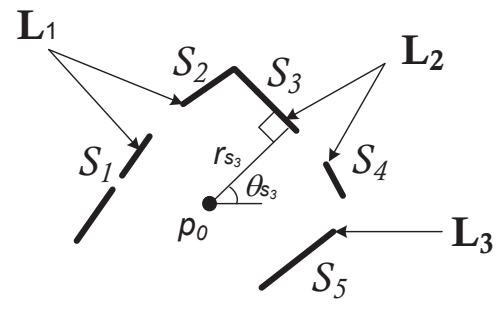

(a)

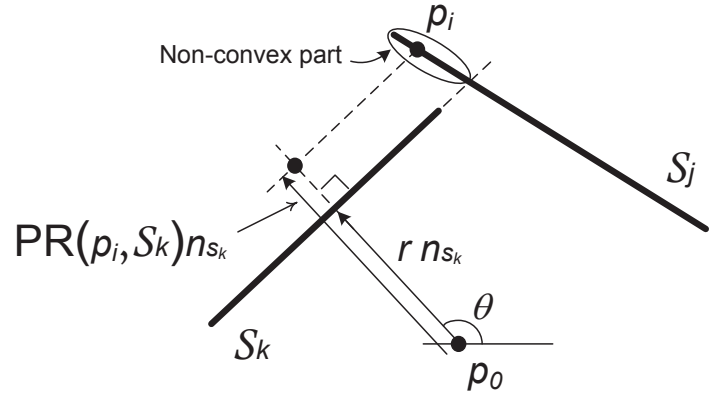

(b)

Figure 3. (a) Linear segments $\mathcal{S}$, linear structures $\mathbf{L}$, and a $\Pi$-structure composed of three linear structures. Linear segments may contain several disconnected components lying on the same line as in the case of $\mathcal{S}_{1}$. Linear structures are composed of aligned linear segments that have similar $\theta$ and $r$. Note that we use the following parametrization $\theta \in[0,180), r \in(-\infty, \infty)$. A valid configuration may contain up to four linear structures. ПI-structure is composed of a triple of linear structures. (b) A pair-wise convexity relationship. A non-convex part of the segment $\mathcal{S}_{j}$ relative to segment $\mathcal{S}_{k}$, given a reference point $p_{0}$, is marked by the ellipse. Corresponding pair-wise relationships between linear segments of a configuration determine the degree of convexity of that configuration, see Definition 1.

for example, to a single linear structure, a pair of perpendicular or parallel structures, three linear structures in a $\Pi$-form as illustrated in Fig. 3(a), or four linear structures arranged in a rectangle.

To identify valid configurations that are likely to correspond to a rectangular enclosure, we propose a rectangularity measure that prefers $\Pi$-structures as described in detail in Sec. 6.2. This measure suppresses configurations coming from a single or a pair of linear structures, thus allowing only three or four sided configurations of linear structures. The proposed rectangularity measure is proportional to the size $l$ of each of the linear segments of a valid configuration. This property allows us to search only maximal cliques ${ }^{\S}$ of the graph, which keeps the number of times the rectangularity measure is computed small.

\subsection{Valid configurations}

To ensure the convexity of a configuration of linear segments, it is sufficient to require that a half plane generated by each segment includes all other segments of the configuration. Additionally, we require that all these half planes contain a single reference point that is, in our case, a candidate point around which we search for a rectangular structure. Actually, we show that pair-wise only constraints suffice to verify the convexity of a configuration that contains the given reference point. We denote the set of linear segments $\mathcal{S}_{k}$ under consideration by $\mathbf{C}=\left\{\mathcal{S}_{1}, \mathcal{S}_{2}, \ldots, \mathcal{S}_{N}\right\}$, which we also call a configuration. We assume that linear segments $\mathcal{S}_{k}$ were already extracted and their attributes $\theta_{\mathcal{S}_{k}}, r_{\mathcal{S}_{k}}$, and $l_{\mathcal{S}_{k}}$ are known. $\theta_{\mathcal{S}_{k}}$ is the orientation angle in degrees, $\left|r_{\mathcal{S}_{k}}\right|$ is the distance from the reference point to the line induced by $\mathcal{S}_{k}$, and $l_{\mathcal{S}_{k}}$ is the size of the linear segment defined as the number of points in the set $\mathcal{S}_{k}$. We use the following parametrization $\theta \in[0,180), r \in(-\infty, \infty)$, which defines any line in a plane. The length of a projection of $p_{i}-p_{0}$ to the unit normal $n_{\mathcal{S}_{k}}=\left(\cos \theta_{\mathcal{S}_{k}}, \sin \theta_{\mathcal{S}_{k}}\right)^{T}$ of a segment $\mathcal{S}_{k}$ is denoted by

$$
\operatorname{PR}\left(p_{i}, \mathcal{S}_{k}\right)=\left(p_{i}-p_{0}\right) \cdot n_{\mathcal{S}_{k}},
$$

which is illustrated in Fig. 3(b). Now we introduce a function $T$, which takes linear segments as arguments

$$
T\left(\mathcal{S}_{k}, \mathcal{S}_{j}\right)=\frac{1}{l_{\mathcal{S}_{j}}} \sum_{p_{i} \in \mathcal{S}_{j}} H\left(\operatorname{sign}\left(r_{\mathcal{S}_{k}}\right)\left(P R\left(p_{i}, \mathcal{S}_{k}\right)-r_{\mathcal{S}_{k}}\right)\right),
$$

where

$$
H(u)= \begin{cases}1, & u>0 \\ 0, & u \leq 0\end{cases}
$$

\footnotetext{
$\S$ Maximal cliques are cliques that are not contained in larger cliques.
} 
and that measures the relative of number of points in segment $\mathcal{S}_{j}$ that are behind the segment $\mathcal{S}_{k}$. These points, marked by an ellipse in Fig. 3(b), are lying behind the segment $\mathcal{S}_{j}$ relative to the given reference point $p_{0}$. Note that $T\left(\mathcal{S}_{k}, \mathcal{S}_{j}\right) \neq T\left(\mathcal{S}_{j}, \mathcal{S}_{k}\right)$. Now, we define the notion of a t-convex configuration, which is an approximately convex configuration, whose convexity can be controlled by the parameter $t$.

Definition 1. Let a configuration $\mathbf{C}=\left\{\mathcal{S}_{i}, i=1, . ., N\right\}$, a reference point $p_{0}$, and a convexity tolerance $t \geq 0$ be given. If for all pairs $j, k=1, . ., N$ with $j \neq k$ both $T\left(\mathcal{S}_{k}, \mathcal{S}_{j}\right) \leq t$ and $T\left(\mathcal{S}_{j}, \mathcal{S}_{k}\right) \leq t$ hold, then $\mathbf{C}$ is a t-convex configuration, denoted by $\mathbf{C}^{t}$.

t-convexity is ensured by pair-wise relations only. It defines the second constraint stated in the beginning of Sec. 6. To be a valid configuration the first constraint needs to be satisfied additionally. This requirement is that angles between linear segments of a t-convex configuration are restricted to be close to either 0 or 90 degrees. Let us define the angle difference between orientations of two segments $\mathcal{S}_{k}, \mathcal{S}_{j}$ as

$$
\Delta \theta_{k, j}=\min \left(\left|\theta_{\mathcal{S}_{k}}-\theta_{\mathcal{S}_{j}}\right|, 180-\left|\theta_{\mathcal{S}_{k}}-\theta_{\mathcal{S}_{j}}\right|\right) .
$$

Note that $0 \leq \Delta \theta_{k, j} \leq 90$.

Definition 2. If for all pairs $\mathcal{S}_{k}, \mathcal{S}_{j} \in \mathbf{C}^{t}, \Delta \theta_{k, j} \leq \alpha$ or $90-\Delta \theta_{k, j} \leq \alpha$, then $\mathbf{C}^{t}$ is a (t, $\left.\alpha\right)$-valid configuration denoted $\mathbf{C}^{t, \alpha}$.

For shortness, we will frequently omit the indices $t$ and $\alpha$, only mentioning that $\mathbf{C}$ is a valid configuration.

As was noted in Sec. 6, we build a graph with linear segments $\mathcal{S}$ as nodes. Given convexity and angle tolerances, $t$ and $\alpha$, an edge between a pair of nodes $\mathcal{S}_{k}$ and $\mathcal{S}_{j}$ is set if both of the following two conditions hold:

1. $\Delta \theta_{k, j} \leq \alpha$ or $90-\Delta \theta_{k, j} \leq \alpha$.

2. $T\left(\mathcal{S}_{k}, \mathcal{S}_{j}\right) \leq t$ and $T\left(\mathcal{S}_{j}, \mathcal{S}_{k}\right) \leq t$.

We will denote such a graph $G^{t, \alpha}$.

Proposition 1. The cliques of a graph $G^{t, \alpha}$ correspond to $(\mathrm{t}, \alpha)$-valid configurations of linear segments.

Proposition 1 directly follows from graph construction and Definitions 1 and 2 . Once a graph $G^{t, \alpha}$ is constructed, the coordinates of the points of the linear segments are no longer required. Only three parameters $\theta, r, l$ are kept for each node.

\subsection{Rectangularity measure}

For a valid configuration $\mathbf{C}=\left\{\mathcal{S}_{k}\right\}$ the joint distribution of $\left(\theta_{\mathcal{S}_{k}}, r_{\mathcal{S}_{k}}\right)$ is clustered around at up to four parametric pairs $(\theta, r)$, each of which corresponds to a particular linear structure $\mathbf{L}_{i}$ of the configuration. This allows us partitioning a valid configuration $\mathbf{C}$ into its four linear structures $\mathbf{L}_{i}$. We propose the following measure of rectangularity of a valid configuration $\mathbf{C}$ with partitioning $\mathbf{L}_{i}, i=1, . ., 4$

$$
R M(\mathbf{C})=l_{L_{1}} l_{L_{2}}\left(l_{L_{3}}+l_{L_{4}}\right)+l_{L_{3}} l_{L_{4}}\left(l_{L_{1}}+l_{L_{2}}\right)
$$

where $l_{L_{i}}$ is the total number of pixels in all segments $\mathcal{S}_{j}$ of a linear structure $\mathbf{L}_{i}$, i.e. $l_{L_{i}}=\sum_{\mathcal{S}_{j} \in \mathbf{L}_{i}} l_{\mathcal{S}_{j}}$. Note that some $\mathbf{L}_{i}, i=1, . ., 4$ of the partitioning can be empty sets with corresponding $l_{L_{i}}=0$. The proposed measure $R M$ has a non-zero value only for configurations with at least three linear structures and it monotonically increases with the size of linear segments. Therefore, it has larger values for configurations of larger size. We use a normalized version of rectangularity measure to be invariant to the size of rectangular structures, which is elaborated later in this section. The rectangularity measure has another important increasing property:

Property 1. Let $\mathbf{C}^{a}$ and $\mathbf{C}^{b}$ be valid configurations with partionings $\mathbf{L}_{i}^{a}, i=1, . ., 4$ and $\mathbf{L}_{i}^{b}, i=1, . ., 4$. If for all $i=1, . ., 4, \mathbf{L}_{i}^{a} \subseteq \mathbf{L}_{i}^{b}$ then $\mathbf{C}^{a} \subseteq \mathbf{C}^{b}$ and $R M\left(\mathbf{C}^{a}\right) \leq R M\left(\mathbf{C}^{b}\right)$.

Given an input set $\mathbf{I}$ of linear segments around a candidate point, we are interested in finding the maximal rectangularity measure $E$ over the set of all possible valid configurations $\boldsymbol{\Omega}(\mathbf{I}), E(\mathbf{I})=\max _{\mathbf{C} \in \boldsymbol{\Omega}(\mathbf{I})} R M(\mathbf{C})$. The 
maximal rectangularity $E$ will serve us as a measure of evidence of a rectangular enclosure we are looking for. Due to the increasing property of the rectangularity measure we need to check only maximal valid configurations $\boldsymbol{\Omega}_{\max } \subseteq \boldsymbol{\Omega}$ that are not contained in larger configurations, i.e.

$$
E(\mathbf{I})=\max _{\mathbf{C} \in \boldsymbol{\Omega}_{\max }} R M(\mathbf{C}) .
$$

Maximal valid configurations correspond to maximal cliques of the graph introduced in the beginning of Sec. 6 . Thus, we consider only maximal cliques of the graph nodes, whose length attributes $l$ are used for computation of rectangularity measures. This considerably lowers the number of times we need to compute Eq. (4). To find maximal cliques of the graph, we use an efficient algorithm introduced in Ref. 19.

The rectangularity measure in Eq. (4) can be cubic in the size of a structure. To be invariant to structure size, we normalize the rectangularity measure by the cubed linear size $D$ (the distance transform at the candidate point) of the structure that was estimated for each candidate point in Sec. 4. Thus, for each candidate point we use $E(\mathbf{I}) / D^{3}$ as the likelihood of existence of rectangular enclosures. Normalization can be also performed in a different way, for example using the values $r_{L_{i}, i=1, . .4}$ of linear structures of an optimal configuration instead of the distance transform $D$. Rectangularity measure is computed for all valid configurations located around candidate points, which is represented by the fifth block in the flow shown in Fig. 1.

\section{EXPERIMENTS}

In this section, we illustrate the application of our method to the detection of remains of approximately rectangular enclosures in remotely sensed images. We used Swiss Topo aerial images and panchromatic images captured by the GeoEye1 satellite. Both types of images are at $0.5 \mathrm{~m}$ resolution. For our experiments, we cropped $600 x 600$ subimages of alpine environment that contain some known remains of livestock enclosures. Walls of enclosures are partially or completely ruined and have low contrasts relative to nearby irrelevant structures and to texture background. Linear segments corresponding to walls of enclosures usually have no more than two pixels width. These factors make detection of livestock enclosures a very challenging task. Examples of livestock enclosures are shown in the left column in Fig. 4. The extracted bright and dark (shadow) ridge features are shown in the second column. The third column shows extracted candidate points (in red). The candidate points were adaptively sampled from a skeleton as described in Sec. 4. In the forth column, we show candidate points with non-zero rectangularity measure computed for valid configurations of linear segments. Valid configurations $\mathbf{C}^{t, \alpha}$ were determined with tolerances $t=0.9$ for the convexity and $\alpha=20^{\circ}$ for the angle. Comparing the third and forth columns of Fig. 4, we can see that lots of candidate points were suppressed since the rectangularity measure has a zero value for valid configurations with less than three linear structures $\mathbf{L}$. The normalized maximal rectangularity measure $E / D^{3}$ (see Sec. 6.2 for details) was computed for all candidate points and used to build the likelihood map shown in the last column of Fig. 4. For each candidate point the normalized maximal rectangularity measure was visualized by a disk color encoded in accordance with the color bar shown in the right of the figure. Note that the color scale is different for each image.

As expected, high likelihood values were generated at positions of known livestock enclosures. Moreover, enclosures far from being rectangular may also be detected as illustrated in the forth row. This is due to the fact that the part of this particular enclosure can be modeled by an approximate rectangle or a polygon with nearly parallel and perpendicular sides. Note, however, that the correspondent likelihood is much lower than for rectangular enclosures in other images. We point out that likelihood values do not depend on illumination conditions and contrast of enclosures, but only on their geometry and their completeness.

Though our approach shows a good potential in detection of livestock enclosures, additional post-validation is required to reduce false detections. The examples in Fig. 4 illustrate the nature of false detections that may be generated. False detections mostly stem from trails, streams or random arrangements of large stones. Our method may generate considerably larger number of false detections in textured areas such as urban or forest areas in remotely sensed images. Therefore, a preprocessing step is required to exclude such areas from the search. See Ref. 9 for an example of such a preprocessing approach.

\footnotetext{
"Of course, walls of an enclosure should be of some minimal contrast to be detected. However, the contrast levels above the minimal contrast are not important.
} 

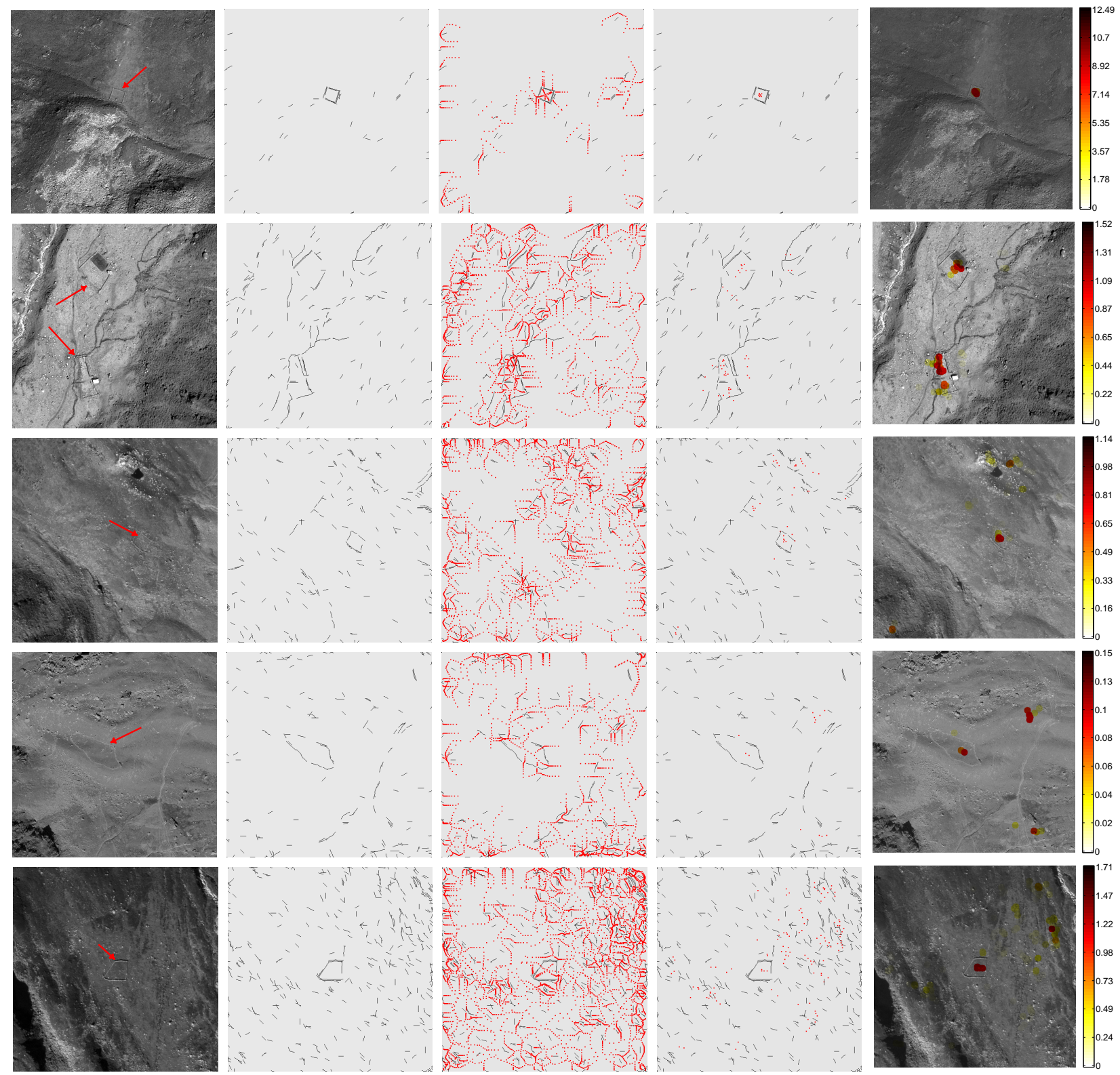

Figure 4. Leftmost column: Images of 600x600 pixels size with livestock enclosures marked by arrows. The first two images are satellite images captured by the GeoEye-1 satellite (C)GeoEye 2011, distributed by e-GEOS), the last three images are aerial SWISSTOPO images. Second column: Ridge features. Third column: Candidate points (in red) superimposed on the map of ridge features. Forth column: Non-zero rectangularity measure computed for valid configurations. Rightmost column: A likelihood map that is a normalized rectangularity measure computed for valid configurations, see Sec. 4 . The values of the likelihood map are encoded by color in accordance with the color bar on the right.

The experiments were carried out using Matlab on a machine with an Intel Core 2 2.83GHz. processor. On average it took 10 seconds to process each of the $600 \times 600$ images including all stages shown in Fig. 1 . Note that the processing time of the last three blocks that are the most time consuming blocks in the diagram in Fig. 1 is proportional to the number of candidate points. Thus, the overall computing time is roughly proportional to the sampling constant $n_{s p}$. For example, halving $n_{s p}$ to 3 reduced the computing time from 10 to 6.5 seconds. We did not, however, analyze the influence of the choice $n_{s p}$ on the detection performance. 


\section{CONCLUSION}

We have introduced a graph based search for structures of approximately rectangular shape in remotely sensed images. Linear features and their orientations were extracted by means of morphological operators. These were further grouped into linear segments using a local Hough transform. Peaks in the Hough plane correspond to linear segments allowing for gaps between aligned linear features. Each linear segment is attributed by a triple of parameters $(\theta, r, l)$ extracted in the Hough plane. A graph is constructed with nodes corresponding to linear segments and with edges encoding spatial constraints between linear segments. An edge between two nodes is given if a corresponding pair of linear segments obeys convexity and angle constraints. Geometrically valid configurations correspond to cliques of the graph. We suggest a rectangularity measure that scores valid configurations of linear segments. Due to the property of the rectangularity measure optimal configurations with the highest score correspond to maximal cliques. This reduces the search space of valid configurations to maximal cliques only. The highest rectangularity score at each candidate point is used as an evidence of approximately rectangular structures. Our approach allows detection of fragmented or incomplete rectangular structures, even if one side is completely missing. It tolerates broken sides, parts of which may be disconnected meeting at angles close to 180 degrees.

Our experiments showed good potential in the detection of livestock enclosures. However, false detections are inevitably generated by our approach that relies only on extracted linear structures and their geometrical relations. False detections may be reduced using other, non-geometrical, features. These, for example, may be spectral properties of regions enclosed by detected configurations of linear segments. We also plan to investigate more general rectangularity measures $R M(\mathbf{C})$ (depending on all the three parameters $\theta, r, l$ of linear structures $\mathbf{L}$ ) that can be learned from the available data. Unfortunately, only a few positive examples of livestock enclosures are currently availablell. On the other hand, there are lots of negative examples available, and we can tolerate relatively high false positive rates. This will guide us to methodologies that allow learning from few examples and are tuned to high sensitivity rates on the expense of false positive rate. False positive rates, however, should be reduced to the lowest possible levels still allowing constrained high sensitivity. Within this framework, we also plan to test the usefulness of various means for artificial generation of positive examples.

\section{Acknowledgments}

This work was funded by the Interreg IV Program "Alpenrhein - Bodensee - Hochrhein" and by the Zukunftskolleg, University of Konstanz. It was also supported by the DFG Research Training Group GK-1042 "Explorative Analysis and Visualization of Large Information Spaces."

\section{REFERENCES}

[1] Lambers, K. and Zingman, I., "Towards detection of archaeological objects in high-resolution remotely sensed images: the Silvretta case study," in [Proc. of Computer Appl. and Quantitative Methods in Archaeology], (2012, in press).

[2] Lambers, K. and Zingman, I., "Texture segmentation as a first step towards archaeological object detection in high-resolution satellite images of the Silvretta alps," in [Archaeological Prospection : Proceedings of the 10th International Conference], Neubauer, W., Trinks, I., Salisbury, R. B., and Einwögerer, C., eds., 327-329, Vienna: Austrian Academy of Sciences (2013).

[3] Lin, C. and Nevatia, R., "Building detection and description from a single intensity image," Computer Vision and Image Understanding 72(2), 101-121 (1998).

[4] Krishnamachari, S. and Chellappa, R., "Delineating buildings by grouping lines with MRFs," IEEE Transactions on Image Processing 5(1), 164-168 (1996).

[5] Kim, T. and Muller, J.-P., "Development of a graph-based approach for building detection," Image Vision Comput. 17(1), 3-14 (1999).

\footnotetext{
${ }^{\|}$We are currently making an effort at collecting additional examples to get a reliable estimate of the performance of our approach.
} 
[6] Croitoru, A. and Doytsher, Y., "Right-angle rooftop polygon extraction in regularised urban areas: Cutting the corners," The Photogrammetric Record 19(108), 311-341 (2004).

[7] Jung, C. R. and Schramm, R., "Rectangle detection based on a windowed Hough transform," in [Proceedings of the Computer Graphics and Image Processing (SIBGRAPI), XVII Brazilian Symposium], 113-120 (2004).

[8] Zingman, I., Saupe, D., and Lambers, K., "Detection of texture and isolated features using alternating morphological filters," in [Mathematical Morphology and Its Applications to Signal and Image Processing], Hendriks, C., Borgefors, G., and Strand, R., eds., Lecture Notes in Computer Science 7883, 440-451, Springer Berlin Heidelberg (2013).

[9] Zingman, I., Saupe, D., and Lambers, K., "Morphological operators for segmentation of high contrast textured regions in remotely sensed imagery," in [Proc. of the IEEE Int. Geoscience and Remote Sensing Symposium], 3451-3454 (July 2012).

[10] Lam, L., Lee, S.-W., and Suen, C. Y., "Thinning methodologies-a comprehensive survey," IEEE Transactions on Pattern Analysis and Machine Intelligence 14(9), 869 - 885 (1992). p.879.

[11] Maurer, C., Qi, R., and Raghavan, V., "A linear time algorithm for computing exact euclidean distance transforms of binary images in arbitrary dimensions," IEEE Transactions on Pattern Analysis and Machine Intelligence 25, 265-270 (Feb. 2003).

[12] Arcelli, C. and di Baja, G. S., "Ridge points in euclidean distance maps," Pattern Recognition Letters 13(4), 237-243 (1992).

[13] Chang, S., Metaxas, D. N., and Axel, L., "Scan-conversion algorithm for ridge point detection on tubular objects.," in [MICCAI (2)], Ellis, R. E. and Peters, T. M., eds., Lecture Notes in Computer Science 2879, 158-165, Springer (2003).

[14] Duda, R. O. and Hart, P. E., "Use of the Hough transformation to detect lines and curves in pictures," Commun. ACM 15, 11-15 (Jan. 1972).

[15] Duda, R. O. and Hart, P. E., [Pattern Classification and Scene Analysis], John Willey \& Sons (1973).

[16] O'Gorman, F. and Clowes, M. B., "Finding picture edges through collinearity of feature points," IEEE Transactions on computers C-25(4), 449-456 (1976).

[17] Dalal, N. and Triggs, B., "Histograms of oriented gradients for human detection," in [Proceedings of the Conference on Computer Vision and Pattern Recognition (CVPR)], 886-893, IEEE Computer Society (2005).

[18] Lowe, D. G., "Distinctive image features from scale-invariant keypoints," Int. J. of Computer Vision 60(2), 91-110 (2004).

[19] Bron, C. and Kerbosch, J., "Algorithm 457: finding all cliques of an undirected graph," Commun. ACM 16, 575-577 (Sept. 1973). 